\title{
On the algebraic K-theory of the Hilbert modular group
}

\author{
MAURICIO BUSTAMANTE \\ LUIS JORGE SÁNCHEZ SALDAÑA
}

\begin{abstract}
We give formulas for the Whitehead groups and the rational K-theory groups of the (integral group ring of the) Hilbert modular group in terms of their maximal finite subgroups.
\end{abstract}

19B28; 19D35

\section{Introduction}

The algebraic K-theory of the integral group ring of a discrete group $G$ is known to encode topological invariants of (high dimensional) manifolds $X$ or spaces whose fundamental group $\pi_{1} X$ is isomorphic to $G$. Examples come in many guises: the obstructions to a finitely dominated space having the homotopy type of a finite CWcomplex, and those to an open smooth manifold being the interior of a compact smooth manifold with boundary, are elements of $\tilde{K}_{0}(\mathbb{Z}[G])\left(\right.$ or $\mathrm{Wh}_{0}(G)$ in our notation below). The obstruction to an $h$-cobordism admitting a product structure $X \times I$ is an element in a quotient of $K_{1}(Z[G])$ denoted here by $\mathrm{Wh}_{1}(G)$ and called the Whitehead group of $G$, and the uniqueness up to isotopy of these product structures on a compact $h$-cobordism has to do with a quotient of $K_{2}(\mathbb{Z}[G])$ named $\mathrm{Wh}_{2}(G)$. In fact an entire sequence of groups $\mathrm{Wh}_{n}(G)$ arising from the higher algebraic K-theory of a group ring can be defined to contain the type of invariants needed to deal with parametrized phenomena in topology, eg pseudoisotopy theory. These groups $\mathrm{Wh}_{n}(G)$ associated to a group $G$ are called Whitehead groups of $G$ and were defined (for larger $n$ ) by Waldhausen [30]. Thus, explicit calculations of algebraic K-theory groups and their associated Whitehead groups $\mathrm{Wh}_{n}$ become relevant in geometric topology.

Unfortunately, such calculations are generally a difficult task to complete, for instance the algebraic K-theory of $\mathbb{Z}$ is not yet known in full for higher degrees. However, sometimes it is possible to approach the lower K-theory and the higher K-theory modulo torsion via a certain generalized homology theory. One instance where this can be done is the integral group ring of the Hilbert modular group $\operatorname{PSL}_{2}\left(\mathcal{O}_{k}\right)$. In fact, we prove the following. 
Theorem 1.1 Let $k$ be a totally real number field of finite degree and let $\mathcal{O}_{k}$ be its ring of algebraic integers. Then for all $q$,

$$
\mathrm{Wh}_{q}\left(\operatorname{PSL}_{2}\left(\mathcal{O}_{k}\right)\right) \cong \bigoplus_{(M)} \mathrm{Wh}_{q}(M),
$$

where the sum runs over the conjugacy classes of the maximal finite subgroups of $\operatorname{PSL}_{2}\left(\mathcal{O}_{k}\right)$.

As for the nonprojective Hilbert modular group $\mathrm{SL}_{2}\left(\mathcal{O}_{k}\right)$, we find that its Whitehead group is determined by $K_{1}\left(\mathbb{Z}\left[\operatorname{PSL}_{2}\left(\mathcal{O}_{k}\right)\right]\right)$.

Theorem 1.2 Let $k$ be a totally real number field of finite degree and let $\mathcal{O}_{k}$ be its ring of algebraic integers. Then

$$
\mathrm{Wh}_{q}\left(\mathrm{SL}_{2}\left(\mathcal{O}_{k}\right)\right) \cong K_{q}\left(\mathbb{Z}\left[\mathrm{PSL}_{2}\left(\mathcal{O}_{k}\right)\right]\right)
$$

for $q \leq 2$.

Although the higher K-theory groups of $\operatorname{PSL}_{2}\left(\mathcal{O}_{k}\right)$ seem to be harder to compute, a lot more can be said about its higher rational K-theory. For a finite group $F$, let

- $r(F)$ denote the number distinct real irreducible representations of $F$,

- $c(F)$ be the number of those real representations that are of complex type,

- $q(F)$ be the number of distinct rational irreducible representations of $F$,

- $k_{p}(F)$ be the number of irreducible representations of $F$ over the $p$-adic numbers $\mathbb{Q}_{p}$, and

- $\quad r_{p}(F)$ be the number of irreducible representations of $F$ over the field with $p$ elements $\mathbb{F}_{p}$.

Denote the rank of an abelian group by rk. Then we have the following.

Theorem 1.3 Let $k$ be a totally real number field of finite degree and let $\mathcal{O}_{k}$ be its ring of algebraic integers. If $G=\operatorname{PSL}_{2}\left(\mathcal{O}_{k}\right)$, then

$\operatorname{rk}\left(K_{q}(\mathbb{Z}[G])\right)-\operatorname{rk}\left(H_{q}(B G ; \mathcal{K}(\mathbb{Z}))\right)$

$$
= \begin{cases}\sum_{(M)} r(M)-m & \text { if } q>2 \text { and } q \equiv 1 \quad \bmod 4, \\ \sum_{(M)} c(M) & \text { if } q>2 \text { and } q \equiv 3 \quad \bmod 4, \\ \sum_{(M)}(r(M)-q(M)) & \text { if } q=1, \\ m-\sum_{(M)}\left[\sum_{p|| M \mid} q(M)\right. & \\ \left.\quad-\left(k_{p}(M)-r_{p}(M)\right)\right] & \text { if } q=-1, \\ 0 & \text { otherwise, }\end{cases}
$$


where $m$ is the number of conjugacy classes of maximal finite subgroups in $G$, the sums range over the conjugacy classes of maximal finite subgroups of $G=\operatorname{PSL}_{2}\left(\mathcal{O}_{k}\right)$, and $H_{q}(B G ; \mathcal{K}(\mathbb{Z}))$ denotes the $q^{\text {th }}$ homology of $B G$ with coefficients in the nonconnective $K$-theory spectrum $\mathcal{K}(\mathbb{Z})$.

The proof of Theorem 1.3, together with Theorem 1.2 lead us to expressions for the classical Whitehead group of the nonprojective Hilbert modular group and the lower reduced K-theory of $\mathrm{SL}_{2}\left(\mathcal{O}_{k}\right)$.

Corollary 1.4 Let $\operatorname{PSL}_{2}\left(\mathcal{O}_{k}\right)^{\mathrm{ab}}$ be the abelianization of $\operatorname{PSL}_{2}\left(\mathcal{O}_{k}\right)$. Then

$$
\mathrm{Wh}_{1}\left(\mathrm{SL}_{2}\left(\mathcal{O}_{k}\right)\right) \simeq \mathrm{Wh}_{1}\left(\mathrm{PSL}_{2}\left(\mathcal{O}_{k}\right)\right) \oplus \operatorname{PSL}_{2}\left(\mathcal{O}_{k}\right)^{\mathrm{ab}} \oplus \mathbb{Z}_{2}
$$

Also,

$$
\begin{aligned}
\mathrm{Wh}_{0}\left(\mathrm{SL}_{2}\left(\mathcal{O}_{k}\right)\right) & \simeq \mathrm{Wh}_{0}\left(\mathrm{PSL}_{2}\left(\mathcal{O}_{k}\right)\right) \oplus \mathbb{Z}, \\
\mathrm{Wh}_{-1}\left(\mathrm{SL}_{2}\left(\mathcal{O}_{k}\right)\right) & \simeq \bigoplus_{(M)} K_{-1}(\mathbb{Z}[M]),
\end{aligned}
$$

where the sum is taken over the conjugacy classes of maximal finite subgroups of $\operatorname{PSL}_{2}\left(\mathcal{O}_{k}\right)$.

The main tool we use to prove our main results is the K-theoretic Farrell-Jones isomorphism conjecture for a group. When this conjecture is verified for a group $\Gamma$, then one can potentially compute the algebraic K-theory of the group ring $\mathbb{Z}[\Gamma]$ by first determining the algebraic K-theory of its virtually cyclic subgroups and the structure of the restricted orbit category $\operatorname{Or}(\Gamma, \mathrm{V} C Y C)$. This will be explained in more detail in Section 2. Then a spectral sequence argument can give rise to the calculation of some of the K-theory groups. This method has proven to be effective in several cases, for example 2- and 3-dimensional crystallographic groups, see Pearson [27], Alves and Ontaneda [1] and Farley and Ortiz [12]; cocompact Fuchsian groups, see Berkove, Juan-Pineda and Pearson [4; 5] and Lück and Stamm [25]; Bianchi groups, see Berkove et al [3]; braid groups, see Juan-Pineda and Millan-López [20; 21]; hyperbolic reflection groups, see Lafont and Ortiz [24]; virtually free groups, see Juan-Pineda et al [19] and Juan-Pineda and Sanchez Saldaña [22]; etc.

The spectral sequence we use in our calculation is the $p$-chain spectral sequence of Davis and Lück which we review in Section 3. This spectral sequence turned out to be convenient due to the structure of the finite subgroups of the Hilbert modular group. We go over this in Section 4. Section 5 is devoted to the proof of Theorems 1.1-1.3. 
Acknowledgements We are grateful to Tom Farrell for suggesting this problem to us and D Juan-Pineda for helpful comments on a preliminary version of this paper. The first author acknowledges the second author for teaching him how to navigate through the somewhat dense literature that surrounds this topic. The second author wishes to thank the Binghamton University for its hospitality during the time in which part of this paper was developed. The first author was supported by a Binghamton University Dissertation Fellowship. The second author was supported by PAPIIT-UNAM grant 105614, CONACYT Research grant 151338 and CONACYT doctoral scholarship.

\section{Preliminaries}

The essential tool in our calculation is the Farrell-Jones isomorphism conjecture. To state this conjecture in a convenient way, we recall some definitions from [9].

From now on we only consider discrete groups. A family of subgroups $\mathcal{F}$ of a group $G$ is always assumed to be closed under conjugation and under taking subgroups. We are specially interested in the following families of subgroups:

- ALL of all subgroups of $G$;

- VCYC of all virtually cyclic subgroups of $G$, ie subgroups which have a (possibly finite) cyclic subgroup of finite index;

- $\mathrm{FBC}$ of finite subgroups and all virtually cyclic subgroups of the form $F \rtimes \mathbb{Z}$ with $F$ finite;

- FIN of all finite subgroups;

- TR consisting of the trivial subgroup.

Note that each family is contained in the previous one.

Definition 2.1 Let $G$ be a group and $\mathcal{F}$ be a family of subgroups. The restricted orbit category $\operatorname{Or}(G, \mathcal{F})$ is the category whose objects are homogenous spaces, also called orbits, $G / H, H \in \mathcal{F}$ and whose morphisms are $G$-maps.

Let $\operatorname{Or}(G)$ denote $\operatorname{Or}(G, \mathrm{ALL})$, and the set of $G$ maps between the orbits $G / H$ and $G / K$ is denoted by $\operatorname{mor}_{G}(G / H, G / K)$.

Note that every element in $\operatorname{mor}_{G}(G / H, G / K)$ is of the form $R_{a}: G / H \rightarrow G / K$ $g H \mapsto g a^{-1} K$, provided $a H a^{-1} \subseteq K$. And that $R_{a}=R_{b}$ if and only if $a b^{-1} \in K$. 
Definition 2.2 Let $G$ be a group and $\mathcal{F}$ a family of subgroups of $G$.

- An $\operatorname{Or}(G, \mathcal{F})$-space (resp. $\operatorname{Or}(G, \mathcal{F})$-spectrum) is a functor from $\operatorname{Or}(G, \mathcal{F})$ to the category of compactly generated topological spaces (resp. spectra); see [9, page 207].

- We denote by $*_{\mathcal{F}}$ the $\operatorname{Or}(G)$-space defined as

$$
*_{\mathcal{F}}(G / H)= \begin{cases}\text { point } & \text { if } H \in \mathcal{F}, \\ \varnothing & \text { otherwise. }\end{cases}
$$

Given a $(G-\mathcal{F})$-space $Z$, ie a $G$-space $Z$ all of whose isotropy groups belong to $\mathcal{F}$, we define the fixed point (contravariant) $\operatorname{Or}(G, \mathcal{F})$-space $\operatorname{map}_{G}(-, Z)$ by $G / H \mapsto \operatorname{map}_{G}(G / H, Z)=Z^{H}$. In particular, if $Z$ is a $G-\mathcal{F} C W$-complex we say that $\operatorname{map}_{G}(-, Z)$ is a free $\operatorname{Or}(G, \mathcal{F}) \mathrm{CW}$-complex.

Remark 2.3 An unreduced homology theory for contravariant $\operatorname{Or}(G, \mathcal{F})$-spaces with coefficients in a covariant $\operatorname{Or}(G, \mathcal{F})$-spectrum can be defined. This homology theory, denoted by $H_{*}^{\operatorname{Or}(G, \mathcal{F})}(X, Y ; \boldsymbol{E})$, is constructed in [9] in order to establish the FarrellJones conjecture. It satisfies the weak homotopy equivalence axiom, ie given a weak homotopy equivalence of pairs of contravariant $\operatorname{Or}(G, \mathcal{F})$-spaces $(f, g):(X, A) \rightarrow$ $(Y, B)$ then the homology groups $H_{p}^{\operatorname{Or}(G, \mathcal{F})}(X, A ; \boldsymbol{E})$ and $H_{p}^{\mathrm{Or}(G, \mathcal{F})}(Y, B ; \boldsymbol{E})$ are isomorphic for all $p \in \mathbb{Z}$.

It can be shown that $H_{n}^{\mathrm{Or}(G)}\left(*_{\mathcal{F}} ; \mathbb{K}\right) \cong \pi_{n}\left(\operatorname{hocolim}_{\mathrm{Or}(G, \mathcal{F})} \mathbb{K}\right)$, where $\mathbb{K}$ denotes the K-theory spectrum defined in [9, Section 2]. Also

$$
H_{n}^{\mathrm{Or}(G)}\left(*_{\mathrm{ALL}} ; \mathbb{K}\right) \cong \pi_{n}\left(\operatorname{hocolim}_{\mathrm{Or}(G)} \mathbb{K}\right) \cong K_{n}(\mathbb{Z}[G])
$$

since $\operatorname{Or}(G)$ has a final object $G / G$ and $\pi_{n}(\mathbb{K}(G / G)) \cong K_{n}(\mathbb{Z}[G])$. It is worth mentioning that in $[9$, Section 2] an $\operatorname{Or}(G, \mathcal{F})$-spectrum $\mathbb{K}$ is defined for every group $G$, in such a way that $\pi_{i}(\mathbb{K}(G / H))=K_{n}(\mathbb{Z} H)$ for all $n \in \mathbb{Z}$.

In their seminal paper, Farrell and Jones [13] established their famous isomorphism conjecture for the K-theory, L-theory and pseudoisotopy functors. Here we consider the K-theoretic version of the conjecture as stated by Davis and Lück [9].

Conjecture 2.4 (Farrell-Jones isomorphism conjecture) For any group $G$, the following assembly map, induced by inclusion of $\operatorname{Or}(G)$-spaces, is an isomorphism

$$
A_{\mathrm{VCYC}, \mathrm{ALL}}: H_{n}^{\mathrm{Or}(G)}\left(*_{\mathrm{VCYC}} ; \mathbb{K}\right) \rightarrow H_{n}^{\mathrm{Or}(G)}\left(*_{\mathrm{ALL}} ; \mathbb{K}\right) \cong K_{n}(\mathbb{Z}[G]) .
$$

Once the Farrell-Jones conjecture has been verified for a group $G$, one can hope to compute $K_{n}(\mathbb{Z}[G])$ using $(*)$. The latter is a generalized homology theory that can be approached, for example, via Mayer-Vietoris sequences, Atiyah-Hirzebruch-type spectral sequences or, as in our case, the $p$-chain spectral sequence. 
The Whitehead groups $\mathrm{Wh}_{n}(G)$ of $G$ appear in this context as follows.

Proposition 2.5 [30, Proposition 15.7] Let $G$ be a group. Then

$$
\mathrm{Wh}_{n}(G) \cong H_{n}^{\mathrm{Or}(G)}\left(*_{\mathrm{ALL}}, *_{\mathrm{TR}} ; \mathbb{K}\right)
$$

for all $n \in \mathbb{Z}$. In fact, the $\mathrm{Wh}_{n}(G)$ fit in a long exact sequence

$$
\cdots \rightarrow H_{n}(B G ; \mathbb{K}(G / 1)) \rightarrow K_{n}(\mathbb{Z}[G]) \rightarrow \mathrm{Wh}_{n}(G) \rightarrow H_{n-1}(B G ; \mathbb{K}(G / 1)) \rightarrow \cdots
$$

where $H_{n}(B G ; \mathbb{K}(G / 1))$ is the classical generalized homology theory with coefficients in the spectrum $\mathbb{K}(G / 1)$ which has as homotopy groups the algebraic $K$-theory of the group ring $\mathbb{Z}[G]$.

When the Farrell-Jones conjecture holds for a group $G$ we obtain the following.

Corollary 2.6 Let $G$ be a group. Suppose that the Farrell-Jones conjecture holds for $G$, then $\mathrm{Wh}_{n}(G)$ is isomorphic to $H_{n}^{\mathrm{Or}(G)}\left(*_{\mathrm{V}_{\mathrm{CYC}}}, *_{\mathrm{TR}} ; \mathbb{K}\right)$ for all $n \in \mathbb{Z}$.

One usually wonders whether a smaller family of subgroups than VCYC would suffice to succeed in the calculation of the Whitehead groups of a group or the K-theory of a group ring. The next two theorems tell us something about this.

Theorem 2.7 [13, Theorem A.10] Let $G$ be a group. Suppose that for any finite by cyclic subgroup $V$ of $G$, the assembly map

$$
A_{\mathrm{FIN}, \mathrm{ALL}}: H_{n}^{\mathrm{Or}(V)}\left(*_{\mathrm{FIN}} ; \mathbb{K}\right) \rightarrow H_{n}^{\mathrm{Or}(V)}\left(*_{\mathrm{ALL}} ; \mathbb{K}\right) \cong K_{n}(\mathbb{Z} V)
$$

is an isomorphism. Then $H_{n}^{\mathrm{Or}(G)}\left(*_{\mathrm{FIN}} ; \mathbb{K}\right) \cong H_{n}^{\mathrm{Or}(G)}\left(*_{\mathrm{FBC}} ; \mathbb{K}\right)$.

Remark 2.8 The obstructions to $A_{\mathrm{FIN}, \mathrm{All}}$ being an isomorphism are the so-called Nil groups. The vanishing of these groups allows one to consider only the family of finite subgroups. Thus under the hypothesis of Theorem 2.7 and using results of [8; $11]$, one can actually compute the Whitehead groups of a group $G$ by considering only its family of finite groups. In other words, if $G$ satisfies the Farrell-Jones conjecture, then

$$
\begin{aligned}
\mathrm{Wh}_{n}(G) & \cong H_{n}^{\mathrm{Or}(G)}\left(*_{\mathrm{ALL}}, *_{\mathrm{TR}} ; \mathbb{K}\right) \\
& \cong H_{n}^{\mathrm{Or}(G)}\left(*_{\mathrm{VCYC}}, *_{\mathrm{TR}} ; \mathbb{K}\right) \\
& \cong H_{n}^{\mathrm{Or}(G)}\left(*_{\mathrm{FBC}}, *_{\mathrm{TR}} ; \mathbb{K}\right) \\
& \cong H_{n}^{\mathrm{Or}(G)}\left(*_{\mathrm{FIN}}, *_{\mathrm{TR}} ; \mathbb{K}\right)
\end{aligned}
$$


Here is one more way in which one can replace the family of subgroups of a group by a smaller family.

Theorem 2.9 [10, Corollary 3.9] Let $\mathcal{F}$ be a family of subgroups of $G$. Denote by $M \mathcal{F} \subset \mathcal{F}$ the subfamily consisting of: all maximal elements in $\mathcal{F}$, the groups in $\mathcal{F}$ which are contained in more than one maximal element, and the groups in $\mathcal{F}$ which are contained in no maximal element of $\mathcal{F}$. Then for all $n$,

$$
H_{n}^{\mathrm{Or}(G)}\left(*_{\mathcal{F}} ; \mathbb{K}\right) \cong H_{n}^{\mathrm{Or}(G)}\left(*_{M \mathcal{F}} ; \mathbb{K}\right)
$$

\section{The $p$-chain spectral sequence}

In this section we establish a special case of the $p$-chain spectral sequence of Davis and Lück [10]. Besides the Farrell-Jones conjecture, this will be the main ingredient in our calculation. The $p$-chain spectral sequence converges to $H^{\operatorname{Or}(\boldsymbol{G}, \mathcal{F})}(X ; \boldsymbol{E})$. By reasons that will become evident in the next section, it will be enough to restrict ourselves to the restricted orbit category $\operatorname{Or}(G$, FIN $)$ and $X$ : the one point $\operatorname{Or}(G, \operatorname{FIN})$-space. This category has the property that every endomorphism is an isomorphism and for each object $G / K, \operatorname{Aut}(G / K)$ acts freely on $\operatorname{mor}_{G}(G / H, G / K)$. Let $\overline{G / H}$ denote the isomorphism class of $G / H$. Note that there is a partial order on the set of isomorphism classes defined by $\overline{G / H} \leq \overline{G / K}$ if $\operatorname{mor}_{G}(G / H, G / K)$ is nonempty. We also write $\overline{G / H}<\overline{G / K}$ if $\overline{G / H} \leq \overline{G / K}$ and $\overline{G / K} \not \leq \overline{G / H}$.

Definition 3.1 Let $G$ be a group and $\mathcal{F}$ a family of subgroups. A sequence of isomorphism classes of objects $c:=\left\{{\bar{G} / H_{0}}_{0}, \overline{G / H}_{1}, \ldots, \overline{G / H}_{p}\right\}$ in $\operatorname{Or}(G, \mathcal{F})$ is called a $p$-chain if

$$
\overline{G / H}_{0}<\overline{G / H}_{1}<\cdots<\overline{G / H}_{p} .
$$

Associated to a $p$-chain $c=\left\{\overline{G / H}_{0}, \overline{G / H}_{1}, \ldots, \overline{G / H}_{p}\right\}$ there are $\operatorname{Aut}\left(G / H_{p}\right)-$ $\operatorname{Aut}\left(G / H_{0}\right)$-spaces $S(c)$ defined by

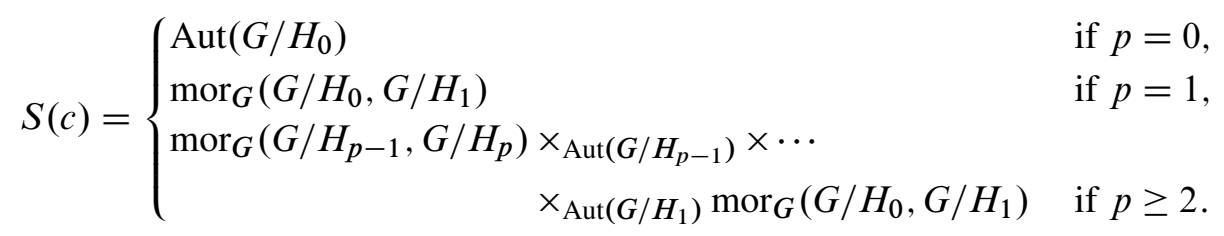

The next result is proven (in more generality) in [9, Lemma 2.11 and Remark 2.13]. 
Theorem 3.2 ( $p$-chain spectral sequence) Let $G$ be a group and let $\mathcal{J} \subset$ FIN be any subfamily of the family of finite subgroups of $G$. Then there is a spectral sequence whose first page is given by

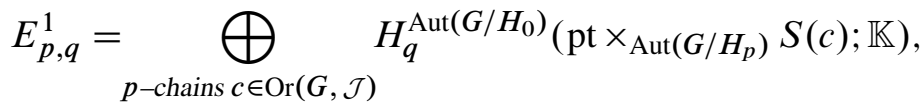

which converges to $H_{p+q}^{\mathrm{Or}(G)}\left(*_{\mathcal{J}} ; \mathbb{K}\right)$.

Remark 3.3 This spectral sequence is very manageable when the length of the $p-$ chains can be controlled. In our case, when $G$ is a Hilbert modular group, we will see that we can work with a specific family of subgroups, for which the restricted orbit category has no $p$-chains for $p \geq 2$. This will make the spectral sequence collapse quickly and explicit calculations can be done.

\section{The Hilbert modular group}

In this section we review the definition and some basic properties of the Hilbert modular group. The results we state without proof in this section can be found, for example, in [14]. For additional information about the Hilbert modular group we refer the reader to $[17 ; 15]$.

A totally real number field $k$ is an algebraic extension of $\mathbb{Q}$ such that all its embeddings $\sigma_{i}: k \rightarrow \mathbb{C}$ have image contained in $\mathbb{R}$. Let $k$ denote a totally real number field of degree $n$ and $\mathcal{O}_{k}$ its ring of algebraic integers. The Hilbert modular group $\operatorname{PSL}_{2}\left(\mathcal{O}_{k}\right)$ is defined to be the quotient of the special linear group of $2 \times 2$ matrices $\operatorname{SL}_{2}\left(\mathcal{O}_{k}\right)$ with entries in $\mathcal{O}_{k}$ by the subgroup $\{I,-I\}$, where $I$ denotes the identity matrix; in other words

$$
\operatorname{PSL}_{2}\left(\mathcal{O}_{k}\right)=\operatorname{SL}_{2}\left(\mathcal{O}_{k}\right) /\{I,-I\} \text {. }
$$

Note that if $k=\mathbb{Q}$, then $\operatorname{PSL}_{2}\left(\mathcal{O}_{k}\right)=\operatorname{PSL}_{2}(\mathbb{Z})$ is nothing but the classical modular group. However $\operatorname{PSL}_{2}\left(\mathcal{O}_{k}\right)$ is not a discrete subgroup of $\operatorname{PSL}_{2}(\mathbb{R})$ if $n \geq 2$. Yet it does act properly and discontinuously on the $n$-fold product $\mathbb{H} \times \cdots \times \mathbb{H}$ of upper half planes, by fractional linear transformations in each of the $n$ factors, via the $n$ different embeddings of $k$ into $\mathbb{R}$. Thus the Hilbert modular group is a discrete subgroup of $\mathrm{PSL}_{2}(\mathbb{R})^{n}=\mathrm{PSL}_{2}(\mathbb{R}) \times \cdots \times \mathrm{PSL}_{2}(\mathbb{R})$. For example, if we let $d$ be a square-free positive integer and $k=\mathbb{Q}(\sqrt{d})$, then there are two embeddings of $k$ into $\mathbb{R}$, namely, for $s, t \in \mathbb{Q}$,

$$
\sigma_{1}: s+t \sqrt{d} \mapsto s+t \sqrt{d} \quad \text { and } \quad \sigma_{2}: s+t \sqrt{d} \mapsto s-t \sqrt{d},
$$


In this case, a matrix $\left(\begin{array}{ll}\alpha & \beta \\ \gamma & \delta\end{array}\right) \in \operatorname{PSL}_{2}\left(O_{k}\right)$ acts on $\left(z_{1}, z_{2}\right) \in \mathbb{H} \times \mathbb{H}$ by

$$
\left(\begin{array}{ll}
\alpha & \beta \\
\gamma & \delta
\end{array}\right)\left(z_{1}, z_{2}\right)=\left(\frac{\sigma_{1}(\alpha) z_{1}+\sigma_{1}(\beta)}{\sigma_{1}(\gamma) z_{1}+\sigma_{1}(\delta)}, \frac{\sigma_{2}(\alpha) z_{2}+\sigma_{2}(\beta)}{\sigma_{2}(\gamma) z_{2}+\sigma_{2}(\delta)}\right) .
$$

The action of $\operatorname{PSL}_{2}\left(\mathcal{O}_{k}\right)$ on $\mathbb{H} \times \cdots \times \mathbb{H}$ is not free. One can detect points with nontrivial isotropy by the following lemma (compare with the analogous situation of $\mathrm{PSL}_{2}(\mathbb{Z})$ acting on $\mathbb{H}$ by Möbius transformations).

Lemma 4.1 Let $h \in \operatorname{PSL}_{2}\left(\mathcal{O}_{k}\right)$, and denote by $\sigma_{i}$ : $\operatorname{PSL}_{2}\left(\mathcal{O}_{k}\right) \hookrightarrow \operatorname{PSL}_{2}(\mathbb{R}), i=$ $1, \ldots, n$, the canonical embeddings of $\operatorname{PSL}_{2}\left(\mathcal{O}_{k}\right)$ into $\operatorname{PSL}_{2}(\mathbb{R})$. Then the following conditions are equivalent:

(1) $\sigma_{1}(h), \ldots, \sigma_{n}(h) \in \mathrm{PSL}_{2}(\mathbb{R})$ are elliptic matrices, ie their traces satisfy

$$
\left[\operatorname{Tr} \sigma_{i}(h)\right]^{2}-4<0 .
$$

(2) $h$ has finite order.

(3) $h$ has a unique fixed point.

Moreover the stabilizer $\Gamma_{z}$ of any point $z \in \mathbb{H} \times \cdots \times \mathbb{H}$ in $\operatorname{PSL}_{2}\left(\mathcal{O}_{k}\right)$ is a finite cyclic group.

For example the matrix $\left(\begin{array}{cc}\sqrt{2}-1 \\ 1 & 0\end{array}\right)$ is an elliptic element of $\operatorname{PSL}_{2}\left(\mathcal{O}_{\mathbb{Q}(\sqrt{2})}\right)$ of order 4 that fixes the point $\frac{\sqrt{2}}{2}((1+i),(-1+i)) \in \mathbb{H} \times \mathbb{H}$.

Remark 4.2 $\mathbb{H} \times \cdots \times \mathbb{H}$ turns out to be a classifying space for proper actions of the Hilbert modular group $\mathrm{PSL}_{2}\left(\mathcal{O}_{k}\right)$, where the fixed point sets of finite subgroups are not only contractible but consist of a single point. This property will simplify the calculation of the K-theory groups enormously.

Perhaps more importantly for our purposes, $\operatorname{PSL}_{2}\left(\mathcal{O}_{k}\right)$ satisfies the conditions $\mathrm{M}, \mathrm{NM}$ and FJ specified in [10, Section 4].

Lemma 4.3 The Hilbert modular group $\operatorname{PSL}_{2}\left(\mathcal{O}_{k}\right)$ satisfies the following three properties:

(M) Every finite subgroup of $\mathrm{PSL}_{2}\left(\mathcal{O}_{k}\right)$ is contained in a unique maximal finite subgroup.

(NM) If $M$ is a maximal finite subgroup of $\operatorname{PSL}_{2}\left(\mathcal{O}_{k}\right)$ then $N(M)=M$, where $N(M)$ denotes the normalizer of $M$ in $\operatorname{PSL}_{2}\left(\mathcal{O}_{k}\right)$.

(FJ) The Farrell-Jones isomorphism conjecture for algebraic K-theory is true for $\operatorname{PSL}_{2}\left(\mathcal{O}_{k}\right)$. 
Proof Note that by Lemma 4.1, if $H$ is a finite subgroup of $\operatorname{PSL}_{2}\left(\mathcal{O}_{k}\right)$ then $H$ is contained in the finite stabilizer $\Gamma_{z}$ of a point $z \in \mathbb{H} \times \cdots \times \mathbb{H} . \Gamma_{z}$ is a finite maximal subgroup of $\operatorname{PSL}_{2}\left(\mathcal{O}_{k}\right)$, for if $F$ is a finite subgroup of $\operatorname{PSL}_{2}\left(\mathcal{O}_{k}\right)$ containing $\Gamma_{z}$, then there must exist $y \in \mathbb{H} \times \cdots \times \mathbb{H}$ such that $f \cdot y=y$ for all $f \in F$. In particular, every element of $\Gamma_{z}$ would fix $y$, and by uniqueness of the fixed points $y=z$, that is to say $f$ is in $\Gamma_{z}$. This proves that $\operatorname{PSL}_{2}\left(\mathcal{O}_{k}\right)$ satisfies property (M).

Let $M$ be a maximal finite subgroup of $\operatorname{PSL}_{2}\left(\mathcal{O}_{k}\right)$. Then by the previous paragraph, $M=\Gamma_{z}$, where $\Gamma_{z}$ is the stabilizer of some point $z \in \mathbb{H} \times \cdots \times \mathbb{H}$. Now let $g$ be in $N(M)$ so that $g f g^{-1} \in M$ for some $f \in M=\Gamma_{z}$. Hence $g f g^{-1} z=z$ which implies that $f g^{-1} z=g^{-1} z$, and by the uniqueness of the fixed points, $g^{-1} z=z$, ie $g \in M$. This proves that $\mathrm{PSL}_{2}\left(\mathcal{O}_{k}\right)$ satisfies the property (NM).

As for property $(\mathrm{FJ})$, note that $\mathrm{PSL}_{2}\left(\mathcal{O}_{k}\right)$ is a lattice in the Lie group $\operatorname{PSL}_{2}(\mathbb{R})^{n}$. The Farrell-Jones conjecture has been proven for this type of group [23].

Remark 4.4 Conditions (M) and (NM) can be interpreted in a somewhat geometric way as follows: let $\mathcal{J}$ be the family of groups $G$ satisfying (FJ) and for which there is a model for $\underline{E} G$ such that every fixed point set by a finite subgroup of $G$ consists of a single point. Then it is clear that if $G \in \mathcal{J}$, then $G$ satisfies (M) and (NM) and (FJ). It is worth noticing that such a family $\mathcal{J}$ is closed under free products.

If a group $G$ has properties (M) and (NM), its only possible virtually cyclic subgroups are very limited.

Lemma 4.5 Let $G$ be a group with properties (M) and (NM), then every infinite virtually cyclic subgroup of $G$ is isomorphic to either $\mathbb{Z}$ or $\mathbb{Z}_{2} * \mathbb{Z}_{2}$.

Proof Every infinite virtually cyclic subgroup $V$ of $G$ fits into an extension

$$
1 \rightarrow F \rightarrow V \rightarrow \Gamma \rightarrow 1,
$$

where $\Gamma$ is either $\mathbb{Z}$ or $\mathbb{Z}_{2} * \mathbb{Z}_{2}$ and $F$ is finite. Since $F$ is normal in $V, V \subset N(F)$, where $N(F)$ is the normalizer of $F$ in $G$. Suppose that $F$ is nontrivial and let $M$ be the unique maximal finite subgroup of $G$ containing $F$. It is clear that $F \subset g g^{-1}$ if $g \in N(F)$. Hence, by uniqueness, $g \in M$. This shows that $N(F) \subset M$, which is finite, contradicting that $V \subset N(F)$. Therefore $F$ is trivial and the conclusion follows.

\section{Whitehead groups and rational K-theory of the Hilbert modular group}

In this section we prove Theorems 1.1, 1.2 and 1.3. 
Proof of Theorem 1.1 Let $G=\operatorname{PSL}_{2}\left(\mathcal{O}_{k}\right)$. Recall that $\mathrm{Wh}_{q}(G)$ is isomorphic to $H_{q}^{\operatorname{Or}(G)}\left(*_{\mathrm{ALL}}, *_{\mathrm{TR}} ; \mathbb{K}\right)$. The first step in our calculation is to verify the Farrell-Jones conjecture for $G$. As mentioned in Lemma 4.3, this follows from [23]. The next step is trying to reduce the family of subgroups as much as possible. In fact, by Lemma 4.5 and the fact that the Nil groups of the integral group rings of $\mathbb{Z}$ and $\mathbb{Z}_{2} * \mathbb{Z}_{2}$ vanish [25, Lemma 2.5], we have that the assembly map

$$
A_{\mathrm{FIN}, \mathrm{ALL}}: H_{q}^{\mathrm{Or}(V)}\left(*_{\mathrm{FIN}} ; \mathbb{K}\right) \rightarrow H_{q}^{\mathrm{Or}(V)}\left(*_{\mathrm{ALL}} ; \mathbb{K}\right)
$$

is an isomorphism. Hence $\mathrm{Wh}_{q}(G)$ can be computed using only the family of finite subgroups of $G$. That is to say

$$
\mathrm{Wh}_{q}(G) \simeq H_{q}^{\mathrm{Or}(G)}\left(*_{\mathrm{FIN}}, *_{\mathrm{TR}} ; \mathbb{K}\right) .
$$

Furthermore, by combining Lemma 4.3(M) and Theorem 2.9, we see that the family of subgroups considered can be reduced further to the subfamily $M$ FIN, where $M$ FIN denotes the family of maximal finite subgroups of $G$ union the trivial subgroup, as defined in Theorem 2.9. Hence

$$
\mathrm{Wh}_{q}(G) \cong H_{q}^{\mathrm{Or}(G)}\left(*_{M \mathrm{FIN}}, *_{\mathrm{TR}} ; \mathbb{K}\right)
$$

Now that we have replaced VCYC by $M$ FIN, we analyze the structure of the $p$-chains in $\operatorname{Or}(G, M$ FIN $)$.

0-chains These are chains of the form $\{\overline{G / H}\}$, where $H \in M$ FIN.

1-chains Here we only have chains of the form $\{\overline{G / 1}, \overline{G / H}\}$. Note that there are no 1 -chains of the form $\{\overline{G / H}, \overline{G / K}\}$ for $H, K \in M$ FIN, $H \neq 1$, because every morphism $G / H \rightarrow G / K$ has to be an isomorphism.

$p$-chains, $p \geq 2$ There are none because every morphism $G / H \rightarrow G / K, H \neq 1$, is an isomorphism.

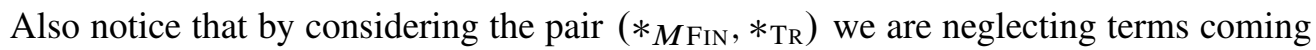
from the 0 -chain $\{G / 1\}$ in the $E^{1}$ page of the $p$-chain spectral sequence for pairs; see Remark 5.1 below. Particularly, no 1-chains have to be considered in the calculation of the Whitehead groups. Thus the spectral sequence will only have contributions from isomorphism classes of orbits of the form $G / H$ with $H \in M$ FIN,$H \neq 1$. Now recall that $\operatorname{Aut}(G / H)=N(H) / H=\{e\}$, where $N(H)$ is the normalizer of $H$ in $G$ and the last equality follows from Lemma 4.3(NM). Also there is a correspondence between 0 -chains in $\operatorname{Or}(G, M$ FIN $)$ and conjugacy classes of maximal finite subgroups in $G$. 
Thus we end up with the following $E^{1}$-term:

$$
\begin{array}{r|lll}
2 & \bigoplus_{(M)} \mathrm{Wh}_{2}(M) & 0 & 0 \\
1 & \bigoplus_{(M)} \mathrm{Wh}_{1}(M) & 0 & 0 \\
0 & \bigoplus_{(M)} \mathrm{Wh}_{0}(M) & 0 & 0 \\
-1 & \bigoplus_{(M)} \mathrm{Wh}_{-1}(M) & 0 & 0 \\
0 & \multicolumn{1}{c}{1} & 2
\end{array}
$$

Therefore the spectral sequence collapses at $E^{1}$ and the result follows.

Remark 5.1 We have used here a relative version of the $p$-chain spectral sequence of a pair of contravariant $\operatorname{Or}(G, \mathcal{F})$-spaces $(X, A)$. In this situation the spectral sequence converges to $H_{*}^{\operatorname{Or}(G, \mathcal{F})}(X, A ; \boldsymbol{E})$, where $\boldsymbol{E}$ is an $\operatorname{Or}(G, \mathcal{F})$-spectrum. The absolute version of the $p$-chain spectral sequence is obtained from a filtration $X_{0} \subset \cdots \subset X_{n}$ of certain $\operatorname{Or}(G, \mathcal{F})$-space which is weakly homotopy equivalent to $X$; see [10]. To obtain the generalization to the relative case, we modify the absolute filtration by one that is relative to a certain $\operatorname{Or}(G, \mathcal{F})$-subspace $\hat{A}$ weakly homotopy equivalent to $A$. It is essentially $\hat{A} \subset \hat{A} \cup X_{0} \subset \cdots \subset \hat{A} \cup X_{i} \subset \cdots \subset X_{n}$. In the case of the pair $\left(*_{M \text { FIN }}, *_{\mathrm{TR}}\right)$, we observe that $*_{M \text { FIN }}(G / 1)-*_{\mathrm{TR}}(G / 1)=\varnothing$. This is why the terms coming from $\{G / 1\}$ do not contribute in the calculation whereas the other terms remain the same.

Proof of Theorem 1.2 We follow the same strategy as in the proof of Theorem 1.1. Let $G=\mathrm{SL}_{2}\left(\mathcal{O}_{k}\right)$. It satisfies the Farrell-Jones conjecture for the same reasons that $\mathrm{PSL}_{2}\left(\mathcal{O}_{k}\right)$ does. Thus we can guarantee that

$$
H_{q}^{\mathrm{Or}(G)}\left(*_{\mathrm{VCYC}} ; \mathbb{K}\right) \cong K_{q}(\mathbb{Z}[G])
$$

for all $q \in \mathbb{Z}$. The idea now is to try to reduce the family of subgroups in order to compute the left side of this equation. Recall that by $[8 ; 11]$ it is always possible to reduce the family from VCYC to $\mathrm{FBC}$; see also Section 2. Hence if we want to replace the family VCYC with FIN it is enough to prove that for any subgroup of $G$ of the form

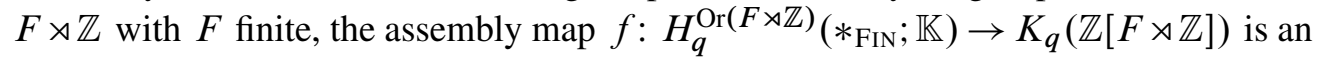
isomorphism. To see this, note that $F \times \mathbb{Z}<F \rtimes \mathbb{Z}$ and the only nontrivial finite-order element that commutes with an element of infinite order is $-I=\left(\begin{array}{cc}-1 & 0 \\ 0 & -1\end{array}\right)$, so every group in the family $\mathrm{FBC}$ is isomorphic to either $\mathbb{Z}$ or $\mathbb{Z}_{2} \times \mathbb{Z}$. By [25, Lemma 2.5] $f$ is an isomorphism for $q \leq 2$ when $F$ is the trivial group or $F \cong \mathbb{Z}_{2}$. Therefore

$$
H_{q}^{\mathrm{Or}(G)}\left(*_{\mathrm{VCYC}} ; \mathbb{K}\right) \cong H_{q}^{\mathrm{Or}(G)}\left(*_{\mathrm{FBC}} ; \mathbb{K}\right) \cong H_{q}^{\mathrm{Or}(G)}\left(*_{\mathrm{FIN}} ; \mathbb{K}\right),
$$

whenever $q \leq 2$. 
We can actually work with a smaller family of subgroups. First note that by the same reasoning as in the proof of Lemma 4.3, every finite maximal subgroup of $G$ is of the form $G_{z}, z \in \underline{E} G=\mathbb{H} \times \cdots \times \mathbb{H}$, the $n$-fold product of upper half-spaces. Hence every finite subgroup different from $\{ \pm I\}$ is contained in a unique finite maximal subgroup. Let $M$ FIN be as defined in Theorem 2.9, and note that every element in MFIN is a maximal finite subgroup, or the trivial subgroup or the group $\{I,-I\}$. By Theorem 2.9 we have

$$
H_{q}^{\mathrm{Or}(G)}\left(*_{\mathrm{FIN}} ; \mathbb{K}\right) \cong H_{q}^{\mathrm{Or}(G)}\left(*_{M \text { FIN }} ; \mathbb{K}\right) .
$$

Now, using the five lemma and the previous reductions of the family of subgroups, it is straightforward to show that, for $q \leq 2$

$$
\mathrm{Wh}_{q}(G) \cong H_{q}^{\mathrm{Or}(G)}\left(*_{\mathrm{ALL}}, *_{\mathrm{TR}} ; \mathbb{K}\right) \cong H_{q}^{\mathrm{Or}(G)}\left(*_{M \mathrm{FIN}}, *_{\mathrm{TR}} ; \mathbb{K}\right) .
$$

We now analyze the $p$-chains that appear in $\operatorname{Or}(G, M$ FIN $)$. In the following, $H$ is a finite maximal subgroup.

0-chains These are chains of the form $\{\overline{G / 1}\},\{\overline{G /\{ \pm I\}}\}$ and $\{\overline{G / H}\}$.

1-chains Chains of the form $\{\overline{G / 1}, \overline{G /\{ \pm I\}}\},\{\overline{G / 1}, \overline{G / H}\}$ and $\{\overline{G /\{ \pm I\}}, \overline{G / H}\}$.

2-chains The only $2-$ chains we can form are of the type $\{\overline{G / 1}, \overline{G /\{ \pm I\}}, \overline{G / H}\}$.

$p$-chains, $p \geq 3$ There are none because for $H, K$ maximal every morphism $G / H \rightarrow$ $G / K$ has to be an isomorphism.

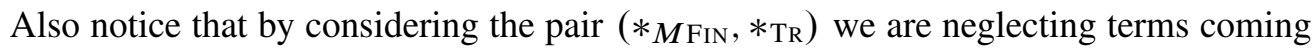
from $p$-chains whose least element is the 0 -chain $\{\overline{G / 1}\}$. Particularly, no 2 -chains have to be considered. Thus the $E^{1}$ term of $p$-chain spectral sequence for pairs will only have contributions from orbits in $\operatorname{Or}\left(G, M \mathrm{FIN}^{0}\right)$, where $M \mathrm{FIN}^{0}:=M \mathrm{FIN}-\mathrm{TR}$. It is clear that $\operatorname{Or}\left(G, M \mathrm{FIN}^{0}\right)$ is equivalent to $\operatorname{Or}\left(\mathrm{PSL}_{2}\left(\mathcal{O}_{k}\right), M\right.$ FIN $)$. Thus the $E^{1}-$ term of the $p$-chain spectral sequence (for pairs) in $\operatorname{Or}(G, M$ FIN) is isomorphic to the $E^{1}$-term of the $p$-chain spectral sequence in $\operatorname{Or}\left(\operatorname{PSL}_{2}\left(\mathcal{O}_{k}\right), M\right.$ FIN $)$. Since the former converges to $\mathrm{Wh}_{*}(G)$ and the latter converges to $K_{*}\left(\mathbb{Z}\left[\mathrm{PSL}_{2}\left(\mathcal{O}_{k}\right)\right]\right)$, the result follows.

Proof of Theorem 1.3 Let $G=\operatorname{PSL}_{2}\left(\mathcal{O}_{k}\right)$. Recall that $M$ FIN from Theorem 2.9 is the family of subgroups of $G$ consisting of all maximal finite subgroups together with the trivial subgroup. In [16, Theorem 5.6], it is proven that for any group $\Gamma$ that satisfies the Farrell-Jones conjecture,

$$
H_{*}^{\mathrm{Or}(\Gamma)}\left(*_{\mathrm{FIN}} ; \mathbb{K}\right) \otimes \mathbb{Q} \cong H_{*}^{\mathrm{Or}(\Gamma)}\left(*_{\mathrm{VCYC}} ; \mathbb{K}\right) \otimes \mathbb{Q} \cong K_{*}(\mathbb{Z}[\Gamma]) \otimes \mathbb{Q}
$$

Now we use Theorem 2.9 to reduce the family from FIN to $M$ FIN. We start analyzing the $p$-chains that appear in $\operatorname{Or}(G, M$ FIN $)$. This has been done already in the proof of 
Theorem 1.1. Note, though, that this time we are not computing the homology of a pair, so we do need to consider 0 -chains and 1-chains (there are no $p$-chains in this category for $p \geq 2$ ). The first page of the $p$-chain spectral sequence is given (after some simplifications, see [4, Proposition 12]) by

$$
\begin{aligned}
& \left.E_{0 q}^{1}=H_{q}(B G ; \mathcal{K}(\mathbb{Z})) \oplus \bigoplus_{(M)} K_{q}(\mathbb{Z}[M])\right), \\
& E_{1 q}^{1}=\bigoplus_{(M)} H_{q}(B M ; \mathcal{K}(\mathbb{Z})), \\
& E_{p q}^{1}=0 \text { for } p \neq 0,1,
\end{aligned}
$$

where $H_{q}(-; \mathcal{K}(\mathbb{Z}))$ refers to the generalized homology theory with coefficients in the Pedersen-Weibel K-theory [28] spectrum and the sum runs over conjugacy classes of maximal finite subgroups. Thus the $p$-chain spectral sequence in this case looks like this, with the following $E^{1}$-term:

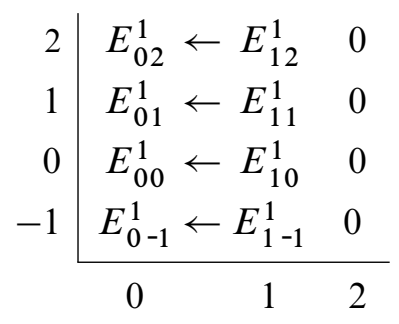

The differentials $d^{1}: E_{1 q}^{1} \rightarrow E_{0 q}^{1}$ are all induced by two types of maps between $p$-chains: those arising from $\{\overline{G / 1}, \overline{G / M}\} \mapsto\{\overline{G / 1}\}$ and those from $\{\overline{G / 1}, \overline{G / M}\} \mapsto$ $\{\overline{G / M}\}$. It is shown in [27, Lemma 3.10] that in the first case the induced map in homology is inclusion whereas the second map induces the classical assembly map $H_{q}(B M ; \mathcal{K}(\mathbb{Z})) \rightarrow K_{q}(\mathbb{Z}[M])$ which in our notation is

$$
A_{\mathrm{TR}, \mathrm{ALL}}: H_{q}^{\mathrm{Or}(M)}\left(*_{\mathrm{TR}} ; \mathbb{K}\right) \rightarrow H_{q}^{\mathrm{Or}(M)}\left(*_{\mathrm{ALL}} ; \mathbb{K}\right) .
$$

In our case this assembly map is known to be rationally injective for all $q$; see [6]. Hence, at least rationally the differentials $d^{1}$ are injective and the spectral sequence (rationally) collapses at $E^{2}$. Then

$$
\operatorname{rk}\left(K_{q}(\mathbb{Z}[G])\right)=\operatorname{rk}\left(E_{0 q}^{\infty}\right)=\operatorname{rk}\left(E_{0 q}^{2}\right) .
$$

Note that $E_{0 q}^{2}$ fits in an exact sequence:

$$
0 \rightarrow E_{1 q}^{1} \otimes \mathbb{Q} \stackrel{d^{1} \otimes \mathrm{id} \mathbb{Q}}{\longrightarrow} E_{0 q}^{1} \otimes \mathbb{Q} \rightarrow E_{0 q}^{2} \otimes \mathbb{Q} \rightarrow 0 .
$$


Hence,

$$
\begin{aligned}
\operatorname{rk}\left(K_{q}(\mathbb{Z}[G])\right) & =\operatorname{rk}\left(E_{0 q}^{2}\right) \\
& =\operatorname{rk}\left(E_{0 q}^{1}\right)-\operatorname{rk}\left(E_{1 q}^{1}\right) \\
& =\sum_{(M)} \operatorname{rk}\left(K_{q}(\mathbb{Z}[M])\right)+\operatorname{rk}\left(H_{q}(B G ; \mathcal{K}(\mathbb{Z}))\right)-\sum_{(M)} \operatorname{rk}\left(H_{q}(B M ; \mathcal{K}(\mathbb{Z}))\right) .
\end{aligned}
$$

Finally, by taking into account that every finite subgroup of the Hilbert modular group $G$ is cyclic, an Atiyah-Hirzebruch spectral sequence calculation shows that

Thus we have

$$
\operatorname{rk}\left(H_{q}(B M ; \mathcal{K}(\mathbb{Z}))\right)= \begin{cases}1 & \text { if } q=0 \text { or } q \equiv 1 \bmod 4, q>2 \\ 0 & \text { otherwise }\end{cases}
$$

$$
\begin{aligned}
\operatorname{rk}\left(K_{q}(\mathbb{Z}[G])\right)-\operatorname{rk}\left(H_{q}(B G ; \mathcal{K}(\mathbb{Z}))\right) \\
= \begin{cases}\sum_{(M)} \operatorname{rk}\left(K_{q}(\mathbb{Z}[M])\right)-m & \text { if } q=0 \text { or } q \equiv 1 \bmod 4, q>2, \\
\sum_{(M)} \operatorname{rk}\left(K_{q}(\mathbb{Z}[M])\right) & \text { otherwise, }\end{cases}
\end{aligned}
$$

where $m$ denotes the number of conjugacy classes of maximal finite subgroups of $G$. By results of Bass [2], Carter [7] and Jahren [18] on the K-theory of finite groups, we have the following equalities:

$$
\operatorname{rk}\left(K_{q}(\mathbb{Z}[M])\right)= \begin{cases}r(M) & \text { if } q>2 \text { and } q \equiv 1 \bmod 4, \\ c(M) & \text { if } q>2 \text { and } q \equiv 3 \bmod 4, \\ r(M)-q(M) & \text { if } q=1, \\ 1 & \text { if } q=0, \\ 1-q(M)+\sum_{p|| M \mid}\left(k_{p}(M)-r_{p}(M)\right) & \text { if } q=-1, \\ 0 & \text { otherwise. }\end{cases}
$$

The result then follows by substituting in the equation $(* *)$.

Proof of Corollary 1.4 Recall that by Theorem 1.2 $\mathrm{Wh}_{1}\left(\mathrm{SL}_{2}\left(\mathcal{O}_{k}\right)\right) \simeq K_{1}\left(\mathrm{PSL}_{2}\left(\mathcal{O}_{k}\right)\right)$. Now notice that the differential of the spectral sequence that appears in the proof of Theorem 1.3 is injective for $q=1$ because the assembly map is injective for $q \leq 1$. This implies that $K_{1}\left(\operatorname{PSL}_{2}\left(\mathcal{O}_{k}\right)\right)=E_{01}^{\infty}=E_{01}^{2}$ and then we obtain an exact sequence

$$
0 \rightarrow E_{11}^{1} \stackrel{d^{1}}{\longrightarrow} E_{01}^{1} \rightarrow K_{1}\left(\mathrm{PSL}_{2}\left(\mathcal{O}_{k}\right)\right) \rightarrow 0 .
$$

It is straightforward (see [4]), with $m$ the number of conjugacy classes of maximal finite subgroups of $\operatorname{PSL}_{2}\left(\mathcal{O}_{k}\right)$, to see that

$$
E_{01}^{1}=\operatorname{PSL}_{2}\left(\mathcal{O}_{k}\right)^{\mathrm{ab}} \oplus \mathbb{Z}_{2} \oplus \bigoplus_{(M)} K_{1}(\mathbb{Z}[M]) \quad \text { and } \quad E_{11}^{1}=\bigoplus_{(M)} M \oplus\left(\mathbb{Z}_{2}\right)^{m} .
$$


Since $M$ is abelian, $K_{1}(\mathbb{Z}[M]) \simeq M \oplus \mathbb{Z}_{2} \oplus \mathrm{Wh}_{1}(M)$ and the image of the assembly map $H_{1}(B M ; \mathcal{K}(\mathbb{Z})) \simeq M \oplus \mathbb{Z}_{2} \rightarrow K_{1}(\mathbb{Z}[M])$ splits for each $M$. This gives us the desired formula for the classical Whitehead group:

$$
\mathrm{Wh}_{1}\left(\mathrm{SL}_{2}\left(\mathcal{O}_{k}\right)\right) \simeq \mathrm{Wh}_{1}\left(\mathrm{PSL}_{2}\left(\mathcal{O}_{k}\right)\right) \oplus \operatorname{PSL}_{2}\left(\mathcal{O}_{k}\right)^{\mathrm{ab}} \oplus \mathbb{Z}_{2}
$$

Similarly we obtain an exact sequence

where

$$
0 \rightarrow E_{10}^{1} \stackrel{d^{1}}{\rightarrow} E_{00}^{1} \rightarrow K_{0}\left(\mathrm{PSL}_{2}\left(\mathcal{O}_{k}\right)\right) \rightarrow 0
$$

$$
E_{10}^{1}=\bigoplus_{(M)} H_{0}(B M ; \mathcal{K}(\mathbb{Z})) \simeq \bigoplus_{(M)} \mathbb{Z} \quad \text { and } \quad E_{00}^{1} \simeq \mathbb{Z} \oplus \bigoplus_{(M)} K_{0}(\mathbb{Z}[M]) .
$$

Note that $K_{0}(\mathbb{Z}[M]) \simeq \mathbb{Z} \oplus \mathrm{Wh}_{0}(M)$ and $\mathrm{Wh}_{0}(M)$ is finite because $M$ is finite. The result then follows by noticing that the assembly map $H_{0}(B M ; \mathcal{K}(\mathbb{Z})) \simeq \mathbb{Z} \rightarrow$ $K_{0}(\mathbb{Z}[M]) \simeq \mathbb{Z} \oplus \mathrm{Wh}_{0}(M)$ is split injective for each $M$.

The same analysis at the $-1^{\text {st }}$ level yields $\mathrm{Wh}_{-1}\left(\mathrm{SL}_{2}\left(\mathcal{O}_{k}\right)\right) \simeq \bigoplus K_{-1}(\mathbb{Z}[M])$. This completes the proof of the corollary.

We conclude this section by working out a concrete example. Let $k=\mathbb{Q}(\sqrt{5})$ the quadratic extension of the rational numbers obtained by adjoining $\sqrt{5}$ to $\mathbb{Q}$. In this case, $\mathrm{PSL}_{2}\left(\mathcal{O}_{k}\right)$ acts on $\mathbb{H} \times \mathbb{H}$ via the embeddings induced by $\sigma_{1}: \sqrt{5} \mapsto \sqrt{5}$ and $\sigma_{2}: \sqrt{5} \mapsto-\sqrt{5}$. By Theorem 1.1, the Whitehead groups of $\operatorname{PSL}_{2}\left(\mathcal{O}_{k}\right)$ are determined by its maximal finite subgroups. Hence our problem reduces to finding all conjugacy classes of maximal finite subgroups. By Lemma 4.3, every maximal finite subgroup of $\operatorname{PSL}_{2}\left(\mathcal{O}_{k}\right)$ appears as a stabilizer of some point in $\mathbb{H} \times \mathbb{H}$. We will say that two fixed points in $\mathbb{H} \times \mathbb{H}$ are inequivalent if their isotropy groups are not conjugate. Thus we have a bijection between conjugacy classes of maximal finite subgroups of $\operatorname{PSL}_{2}\left(\mathcal{O}_{k}\right)$ and the number of inequivalent fixed points in $\mathbb{H} \times \mathbb{H}$. The problem of finding the number of fixed points of the action of the Hilbert modular group of a quadratic number field has been addressed [29]. We sketch here the way to proceed: since only elliptic elements of $\operatorname{PSL}_{2}\left(\mathcal{O}_{k}\right)$ can have fix points, then the trace of such an element must satisfy

$$
\left[\operatorname{Tr} \sigma_{i}(h)\right]^{2}-4<0, \quad i=1,2 .
$$

Also, every elliptic matrix is conjugate (in $\left.\mathrm{PSL}_{2}(\mathbb{C})\right)$ to one of the form $\left(\begin{array}{ll}\omega & 0 \\ 0 & \frac{\omega}{\omega}\end{array}\right)$, where $\omega$ is a primitive root of unity. Thus, in our case, each of these traces should be an algebraic integer of $\mathbb{Q}(\sqrt{5})$, ie an element of $\mathbb{Z}[(1+\sqrt{5}) / 2]$. The only possibilities are $0, \pm 1$ and $\pm(1 \pm \sqrt{5}) / 2$, corresponding to a $4^{\text {th }}, 6^{\text {th }}$ and $10^{\text {th }}$ root of unity, respectively. Thus in $\operatorname{PSL}_{2}\left(\mathcal{O}_{k}\right)$ we will only have elliptic elements of order 2, 3 and 5; consequently the only finite subgroups that can appear will be isomorphic to $\mathbb{Z}_{2}, \mathbb{Z}_{3}$ and $\mathbb{Z}_{5}$. The 
number of conjugacy classes of these maximal finite subgroups is calculated in [29]: each of these groups has exactly two conjugacy classes in $\operatorname{PSL}_{2}\left(\mathcal{O}_{k}\right)$. Therefore, by Theorem 1.1 we have

$$
\mathrm{Wh}_{q}\left(\operatorname{PSL}_{2}\left(\mathcal{O}_{\mathbb{Q}(\sqrt{5})}\right)\right)=\mathrm{Wh}_{q}\left(\mathbb{Z}_{2}\right)^{2} \oplus \mathrm{Wh}_{q}\left(\mathbb{Z}_{3}\right)^{2} \oplus \mathrm{Wh}_{q}\left(\mathbb{Z}_{5}\right)^{2} .
$$

In the special case of the classical Whithead group, ie $q=1$, it is known [26] that $\mathrm{Wh}\left(\mathbb{Z}_{2}\right) \simeq \mathrm{Wh}\left(\mathbb{Z}_{3}\right) \simeq 0$ and $\mathrm{Wh}\left(\mathbb{Z}_{5}\right) \simeq \mathbb{Z}$. Therefore

$$
\mathrm{Wh}\left(\operatorname{PSL}_{2}\left(\mathcal{O}_{\mathbb{Q}(\sqrt{5})}\right)\right) \simeq \mathbb{Z} \oplus \mathbb{Z}
$$

Also, by Corollary 1.4 we have

$$
\mathrm{Wh}\left(\mathrm{SL}_{2}\left(\mathcal{O}_{\mathbb{Q}(\sqrt{5})}\right)\right) \simeq \mathbb{Z} \oplus \mathbb{Z} \oplus \mathbb{Z}_{2},
$$

since $\operatorname{PSL}_{2}\left(\mathcal{O}_{\mathbb{Q}(\sqrt{5})}\right)$ is a perfect group. Finally for the higher K-theory we obtain (here $G=\operatorname{PSL}_{2}\left(\mathcal{O}_{\mathbb{Q}(\sqrt{5})}\right)$ ):

$\operatorname{rk} K_{q}(\mathbb{Z}[G])-\operatorname{rk} H_{q}(B G ; \mathcal{K}(\mathbb{Z}))$

$$
\begin{aligned}
& = \begin{cases}2 r\left(\mathbb{Z}_{2}\right)+2 r\left(\mathbb{Z}_{3}\right)+2 r\left(\mathbb{Z}_{5}\right) & \text { if } q>2 \text { and } q \equiv 1 \bmod 4, \\
2 c\left(\mathbb{Z}_{2}\right)+2 c\left(\mathbb{Z}_{3}\right)+2 c\left(\mathbb{Z}_{5}\right) & \text { if } q>2 \text { and } q \equiv 3 \bmod 4, \\
2\left(r\left(\mathbb{Z}_{2}\right)-q\left(\mathbb{Z}_{2}\right)\right)+2\left(r\left(\mathbb{Z}_{3}\right)-q\left(\mathbb{Z}_{3}\right)\right) & \\
+2\left(r\left(\mathbb{Z}_{5}\right)-q\left(\mathbb{Z}_{5}\right)\right) & \text { if } q=1 ;\end{cases} \\
& = \begin{cases}4+4+6 & \text { if } q>2 \text { and } q \equiv 1 \bmod 4, \\
0+2+4 & \text { if } q>2 \text { and } q \equiv 3 \bmod 4, \\
0+0+2 & \text { if } q=1\end{cases} \\
& = \begin{cases}14 & \text { if } q>2 \text { and } q \equiv 1 \bmod 4, \\
6 & \text { if } q>2 \text { and } q \equiv 3 \bmod 4, \\
2 & \text { if } q=1 .\end{cases}
\end{aligned}
$$

Remark 5.2 It is worth noticing that the formula of Corollary 1.4 also provides a calculation for the Whitehead group of $\mathrm{SL}_{2}(\mathbb{Z})$ :

$$
\begin{aligned}
\mathrm{Wh}_{1}\left(\mathrm{SL}_{2}(\mathbb{Z})\right) & \simeq \mathrm{Wh}_{1}\left(\mathrm{PSL}_{2}(\mathbb{Z})\right) \oplus \mathrm{PSL}_{2}(\mathbb{Z})^{\mathrm{ab}} \oplus \mathbb{Z}_{2} \\
& \simeq \mathrm{Wh}_{1}\left(\mathbb{Z}_{2} * \mathbb{Z}_{3}\right) \oplus \mathbb{Z}_{6} \oplus \mathbb{Z}_{2} \\
& \simeq \mathbb{Z}_{6} \oplus \mathbb{Z}_{2},
\end{aligned}
$$

where we have used the fact that $\mathrm{Wh}_{1}\left(\mathbb{Z}_{2} * \mathbb{Z}_{3}\right) \simeq \mathrm{Wh}_{1}\left(\mathbb{Z}_{2}\right) \oplus \mathrm{Wh}_{1}\left(\mathbb{Z}_{3}\right) \simeq 0$.

The algebraic K-theory of the modular group $\mathrm{PSL}_{2}(\mathbb{Z})$ has also been studied in $[19$, Section 3.2] and [8, Section 3.4]. 


\section{References}

[1] A Alves, P Ontaneda, A formula for the Whitehead group of a three-dimensional crystallographic group, Topology 45 (2006) 1-25 MR2170492

[2] H Bass, The Dirichlet unit theorem, induced characters, and Whitehead groups of finite groups, Topology 4 (1965) 391-410 MR0193120

[3] E Berkove, F T Farrell, D Juan-Pineda, K Pearson, The Farrell-Jones isomorphism conjecture for finite covolume hyperbolic actions and the algebraic K-theory of Bianchi groups, Trans. Amer. Math. Soc. 352 (2000) 5689-5702 MR1694279

[4] E Berkove, D Juan-Pineda, K Pearson, The lower algebraic K-theory of Fuchsian groups, Comment. Math. Helv. 76 (2001) 339-352 MR1839350

[5] E Berkove, D Juan-Pineda, K Pearson, A geometric approach to the lower algebraic K-theory of Fuchsian groups, Topology Appl. 119 (2002) 269-277 MR1888672

[6] M Bökstedt, W C Hsiang, I Madsen, The cyclotomic trace and algebraic K-theory of spaces, Invent. Math. 111 (1993) 465-539 MR1202133

[7] D W Carter, Lower K-theory of finite groups, Comm. Algebra 8 (1980) 1927-1937 MR590500

[8] J F Davis, Q Khan, A Ranicki, Algebraic K-theory over the infinite dihedral group: an algebraic approach, Algebr. Geom. Topol. 11 (2011) 2391-2436 MR2835234

[9] J F Davis, W Lück, Spaces over a category and assembly maps in isomorphism conjectures in K- and L-theory, K-Theory 15 (1998) 201-252 MR1659969

[10] J F Davis, W Lück, The p-chain spectral sequence, K-Theory 30 (2003) 71-104 MR2061848

[11] J F Davis, F Quinn, H Reich, Algebraic K-theory over the infinite dihedral group: a controlled topology approach, J. Topol. 4 (2011) 505-528 MR2832565

[12] DS Farley, I J Ortiz, Algebraic K-theory of crystallographic groups: the threedimensional splitting case, Lecture Notes in Mathematics 2113, Springer, Cham (2014) MR3308161

[13] F T Farrell, L E Jones, Isomorphism conjectures in algebraic K-theory, J. Amer. Math. Soc. 6 (1993) 249-297 MR1179537

[14] E Freitag, Hilbert modular forms, Springer-Verlag, Berlin (1990) MR1050763

[15] G van der Geer, Hilbert modular surfaces, Ergeb. Math. Grenzgeb. 16, Springer, Berlin (1988) MR930101

[16] J Grunewald, The behavior of Nil-groups under localization and the relative assembly map, Topology 47 (2008) 160-202 MR2414359

[17] F E P Hirzebruch, Hilbert modular surfaces, Enseignement Math. 19 (1973) 183-281 MR0393045 
[18] B Jahren, Involutions on the rational $K$-theory of group rings of finite groups, from: "Alpine perspectives on algebraic topology", (C Ausoni, K Hess, J Scherer, editors), Contemp. Math. 504, Amer. Math. Soc. (2009) 189-202 MR2581909

[19] D Juan-Pineda, J-F Lafont, S Millan-Vossler, S Pallekonda, Algebraic K-theory of virtually free groups, Proc. Roy. Soc. Edinburgh Sect. A 141 (2011) 1295-1316 MR2855898

[20] D Juan-Pineda, S Millan-López, Invariants associated to the pure braid group of the sphere, Bol. Soc. Mat. Mexicana 12 (2006) 27-32 MR2301740

[21] D Juan-Pineda, S Millan-López, The Whitehead group and the lower algebraic $K$ theory of braid groups on $\mathbb{S}^{2}$ and $\mathbb{R P}^{2}$, Algebr. Geom. Topol. 10 (2010) 1887-1903 MR2728479

[22] D Juan-Pineda, L J Sánchez Saldaña, On the ranks of the algebraic K-theory of hyperbolic groups, Bol. Soc. Mat. Mex. 20 (2014) 277-285 MR3264616

[23] H Kammeyer, W Lück, H Rüping, The Farrell-Jones conjecture for arbitrary lattices in virtually connected Lie groups, Geom. Topol. 20 (2016) 1275-1287 MR3523058

[24] J-F Lafont, I J Ortiz, Lower algebraic K-theory of hyperbolic 3-simplex reflection groups, Comment. Math. Helv. 84 (2009) 297-337 MR2495796

[25] W Lück, R Stamm, Computations of $K$ - and L-theory of cocompact planar groups, K-Theory 21 (2000) 249-292 MR1803230

[26] R Oliver, Whitehead groups of finite groups, London Mathematical Society Lecture Note Series 132, Cambridge Univ. Press (1988) MR933091

[27] K Pearson, Algebraic K-theory of two-dimensional crystallographic groups, K-Theory 14 (1998) 265-280 MR1633509

[28] E K Pedersen, C A Weibel, A nonconnective delooping of algebraic K-theory, from: "Algebraic and geometric topology", Lecture Notes in Math. 1126, Springer, Berlin (1985) 166-181

[29] A Prestel, Die elliptischen Fixpunkte der Hilbertschen Modulgruppen, Math. Ann. 177 (1968) 181-209 MR0228439

[30] F Waldhausen, Algebraic K-theory of generalized free products, II, Ann. of Math. (2) 108 (1978) 205-256 MR0498808

Department of Mathematical Sciences, Binghamton University

4400 Vestal Pkwy E, Binghamton, NY 13902, United States

Centro de Ciencias Matemáticas, UNAM, Campus Morelia

58190 Michoacán, CP, Mexico

bustamante@math.binghamton.edu, luisjorge@matmor.unam.mx

Received: 28 March $2015 \quad$ Revised: 3 November 2015 
